\title{
Almost Global Existence of Solutions to the Kadomtsev-Petviashvili Equations
}

\author{
By \\ Nakao Hayashi ${ }^{1}$, Pavel I. Naumkin ${ }^{2}$ and Tomoyuki NiIzATo ${ }^{3}$ \\ (Osaka University ${ }^{1,3}$, Japan and $\mathrm{UNAM}^{2}$, Mexico)
}

\begin{abstract}
We consider the Cauchy problem for the Kadomtsev-Petviashvili equations $u_{t}+u_{x x x}+\sigma \partial_{x}^{-1} u_{y y}=-\left(u^{2}\right)_{x}, \quad(x, y) \in \boldsymbol{R}^{2}, \quad t \in \boldsymbol{R}, \quad u(0, x, y)=u_{0}(x, y), \quad(x, y) \in \boldsymbol{R}^{2}$, where $\sigma=1$ or $\sigma=-1, \partial_{x}^{-1}=\int_{-\infty}^{x} d x^{\prime}$. We prove that the maximal existence time $T$ is estimated from below as $T \geq \exp (C / \varepsilon)$, where $\varepsilon$ denotes the size of the initial data, $C>0$ is a constant.

Key Words and Phrases. Kadomtsev-Petviashvili equations, Almost global existence.
\end{abstract}

2010 Mathematics Subject Classification Numbers. 35Q53.

\section{Introduction}

We study the Cauchy problem for the Kadomtsev-Petviashvili equations

$$
\begin{cases}u_{t}+u_{x x x}+\sigma \partial_{x}^{-1} u_{y y}=-\partial_{x}\left(u^{2}\right), & (x, y) \in \boldsymbol{R}^{2}, t \in \boldsymbol{R}, \\ u(0, x, y)=u_{0}(x, y), & (x, y) \in \boldsymbol{R}^{2}\end{cases}
$$

where $\sigma= \pm 1$ and $\partial_{x}^{-1}=\int_{-\infty}^{x} d x^{\prime}$. When $\sigma=-1,(1.1)$ is known as the KPI equation, while $\sigma=1$ is known as KPII equation. The KP equation models the propagation along the $x$-axis of nonlinear dispersive long waves on the surface of a fluid, when the variation along the $y$-axis proceeds slowly (see [5]). Our purpose in this paper is to prove an almost global in time existence of solutions to (1.1).

The Cauchy problem for KPI and KPII equations was studied by many authors. Ukai [12] has shown the local well-posedness of the Cauchy problem in $\boldsymbol{H}^{s}, s \geq 3$, while Iório and Nunes [4] obtained the local well-posedness in $\boldsymbol{H}^{s}, s>2$ for a class of generalized KP equations. On the other hand, Bourgain [2] has proved the global well-posedness in $\boldsymbol{H}^{s}, s \geq 0$ for the KPII case with periodic initial data in addition to the Cauchy problem. Recently some rigorous results were appeared for the generalized KP equation

$$
\begin{cases}u_{t}+u_{x x x}+\sigma \partial_{x}^{-1} u_{y y}=-\partial_{x}\left(u^{\rho}\right), & (x, y) \in \boldsymbol{R}^{2}, t \in \boldsymbol{R}, \\ u(0, x, y)=u_{0}(x, y), & (x, y) \in \boldsymbol{R}^{2}\end{cases}
$$


In paper [7] it was shown that certain solutions of the generalized KP equation (1.2) with $\sigma=-1, \rho \geq 5$ can not remain in the Sobolev space $\boldsymbol{H}^{1}$ for all time. This blow-up phenomena is essentially due to the transverse effects (namely, the norm of the derivative $\left\|\partial_{y} u(t)\right\|_{L^{2}}$ tends to infinity in a finite time). Some results concerning existence of the solitary wave solutions for the generalized KPI equation (1.2) with $\sigma=-1$ were obtained in paper [1], where it was proved that equation (1.2) with $\sigma=-1$ does not possess any nontrivial solitary wave solution if $\rho \geq 5$ and in the case $2 \leq \rho<5$ nontrivial solitary waves do exist. Tom proved in [10] the existence of global solutions to the Cauchy problem for the generalized KP equation (1.1) with $\sigma=-1,2 \leq \rho \leq 5$ in the natural energy space associated to the KP equations. Indeed, the two conservation laws are valid (see [7])

$$
\frac{d}{d t}\|u\|_{L^{2}}^{2}=0
$$

and

$$
\frac{d}{d t}\left(-\frac{1}{2}\left\|\partial_{x} u\right\|_{L^{2}}^{2}+\frac{\sigma}{2}\left\|\partial_{x}^{-1} \partial_{y} u\right\|_{L^{2}}^{2}+\frac{1}{\rho+1}\|u\|_{L^{\rho+1}}^{\rho+1}\right)=0 .
$$

In particular, the existence of global solutions was proved without any size restriction on the data for the case of $2 \leq p<7 / 3$. However the uniqueness of such solutions is an open problem. Global existence of unique solutions to (1.2) for $\rho \in N, \rho \geq 3$ and time decay estimates were studied in [3] and [6].

To state our results precisely we introduce Notation and Function Spaces. As usually, we denote the Lebesgue space by $\boldsymbol{L}^{p}=\left\{\phi \in \boldsymbol{S}^{\prime} ;\|\phi\|_{\boldsymbol{L}^{p}}<\infty\right\}$, where the norm $\|\phi\|_{\boldsymbol{L}^{p}}=\left(\iint_{\boldsymbol{R}^{2}}|\phi(x, y)|^{p} d x d y\right)^{1 / p}$ for $1 \leq p<\infty$ and $\|\phi\|_{\boldsymbol{L}^{\infty}}=$ $\sup _{(x, y) \in \boldsymbol{R}^{2}}|\phi(x, y)|$ for $p=\infty$. The weighted Sobolev space is

$$
\boldsymbol{H}_{p}^{m, s}=\left\{\varphi \in \boldsymbol{S}^{\prime} ;\|\phi\|_{\boldsymbol{H}_{p}^{m, s}}=\left\|\left(1+x^{2}+y^{2}\right)^{s / 2}(1-\Delta)^{m / 2} \phi(x, y)\right\|_{\boldsymbol{L}^{p}}<\infty\right\},
$$

$m, s \in \boldsymbol{R}, \quad 1 \leq p \leq \infty, \quad \Delta=\partial_{x}^{2}+\partial_{y}^{2}$. We also use the following notations $\boldsymbol{H}^{m, s}=\boldsymbol{H}_{2}^{m, s}$. The usual Sobolev space is $\boldsymbol{H}^{m}=\boldsymbol{H}^{m, 0}$, so the index 0 we usually omit if it does not cause a confusion. Let $\boldsymbol{C}(\boldsymbol{I} ; \boldsymbol{B})$ be the space of continuous functions from an interval $\boldsymbol{I}$ to a Banach space $\boldsymbol{B}$. Different positive constants might be denoted by the same letter $C$. By $\mathscr{F} \phi$ or $\hat{\phi}$ we denote the Fourier transform of the function $\phi$

$$
\hat{\phi}(\xi, \eta)=\frac{1}{2 \pi} \iint_{\boldsymbol{R}^{2}} e^{-i x \xi-i y \eta} \phi(x, y) d x d y .
$$

Then the inverse Fourier transform $\mathscr{F}^{-1}$ is given by

$$
\mathscr{F}^{-1} \phi=\frac{1}{2 \pi} \iint_{\boldsymbol{R}^{2}} e^{i x \xi+i y \eta} \phi(\xi, \eta) d \xi d \eta .
$$


Denote by $\mathscr{U}(t)=\mathscr{F}^{-1} e^{i t\left(\xi^{3}-\sigma \eta^{2} / \xi\right)} \mathscr{F}$ the free KP evolution group. The integral equation associated with (1.1) can be written as follows

$$
u(t)=\mathscr{U}(t) u_{0}-\int_{0}^{t} \mathscr{U}(t-s) \partial_{x}\left(u^{2}\right) d s .
$$

To get the $\boldsymbol{L}^{\infty}$-estimates of the solutions we use the following operators $\mathscr{J}_{x}=\mathscr{U}(t) x \mathscr{U}(-t)=x-t\left(3 \partial_{x}^{2}-\sigma \partial_{x}^{-2} \partial_{y}^{2}\right)$ and $\mathscr{J}_{y}=\mathscr{U}(t) y \mathscr{U}(-t)=y-2 \sigma t \partial_{x}^{-1} \partial_{y}$ (see [3]). Since the operator $\mathscr{J}_{x}$ does not work well for the nonlinear term of $\mathrm{KP}$ equation, we also introduce the operator

$$
\mathscr{P}=x \partial_{x}+2 y \partial_{y}+3 t \partial_{t}=\mathscr{J}_{x} \partial_{x}+2 \mathscr{J}_{y} \partial_{y}+3 t \mathscr{L},
$$

where $\mathscr{L}=\partial_{t}+\partial_{x}^{3}+\sigma \partial_{x}^{-1} \partial_{y}^{2}$ is the linear part of KP equation. The operators $\mathscr{J}_{x}, \mathscr{J}_{y}, \mathscr{P}$ satisfy the following commutator relations

$$
\begin{array}{ll}
{\left[\mathscr{L}, \mathscr{J}_{x}\right]=\left[\mathscr{L}, \mathscr{J}_{y}\right]=\left[\mathscr{J}_{x}, \partial_{y}\right]=\left[\mathscr{J}_{y}, \partial_{x}\right]=0, \quad[\mathscr{L}, \mathscr{P}]=3 \mathscr{L},} \\
{\left[\mathscr{J}_{x}, \partial_{x}\right]=\left[\mathscr{J}_{y}, \partial_{y}\right]=-1, \quad\left[\mathscr{P}, \partial_{x}\right]=-\partial_{x}, \quad\left[\mathscr{P}, \partial_{y}\right]=-2 \partial_{y} .}
\end{array}
$$

We define the function space

$$
\boldsymbol{X}_{T}=\left\{\phi \in \boldsymbol{C}\left([0, T] ; \boldsymbol{L}^{2}\left(\boldsymbol{R}^{2}\right)\right):\|\phi\|_{\boldsymbol{X}_{T}}<\infty\right\},
$$

where the norm

$$
\|\phi\|_{\boldsymbol{X}_{T}}=\sup _{t \in[0, T]}\left(\left\|\partial_{y} \partial_{x}^{-1} \phi\right\|_{\boldsymbol{H}^{1}}+\|\phi\|_{\boldsymbol{H}^{1}}+\left\|\partial_{x}^{3} \phi\right\|_{\boldsymbol{L}^{2}}+\left\|\mathscr{J}_{y}^{2} \partial_{x} \phi\right\|_{\boldsymbol{L}^{2}}+\left\|\mathscr{J}_{x} \partial_{x} \phi\right\|_{\boldsymbol{L}^{2}}\right) .
$$

For the initial data $u_{0}$ we define

$$
\boldsymbol{X}_{0}=\left\{\phi \in \boldsymbol{L}^{2}\left(\boldsymbol{R}^{2}\right):\|\phi\|_{\boldsymbol{X}_{0}}<\infty\right\},
$$

where the norm

$$
\|\phi\|_{\boldsymbol{X}_{0}}=\left\|\partial_{y} \partial_{x}^{-1} \phi\right\|_{\boldsymbol{H}^{1}}+\|\phi\|_{\boldsymbol{H}^{1}}+\left\|\partial_{x}^{3} \phi\right\|_{\boldsymbol{L}^{2}}+\left\|y^{2} \partial_{x} \phi\right\|_{\boldsymbol{L}^{2}}+\left\|x \partial_{x} \phi\right\|_{\boldsymbol{L}^{2}}
$$

We denote a maximal existence time by

$$
T^{*}=\sup \left\{T>0 ;\|u\|_{\boldsymbol{X}_{T}}<\infty\right\} .
$$

Theorem 1. Let $u_{0} \in \boldsymbol{X}_{0}$ and the norm $\left\|u_{0}\right\|_{\boldsymbol{X}_{0}}=\varepsilon_{0}$. Suppose that the local solution $u \in \boldsymbol{C}\left([0, T] ; \boldsymbol{L}^{2}\left(\boldsymbol{R}^{2}\right)\right)$ of the Cauchy problem for the KadomtsevPetviashvili equation (1.1) exists on a time interval $[0, T]$ and the norm $\|u\|_{X_{T}}<\infty$. Then there exist positive constants $\varepsilon_{0}$ and $B$, such that the following estimate for the maximal existence time is true

$$
T^{*} \geq \exp \left(\frac{B}{\varepsilon}\right)
$$

for any $0<\varepsilon \leq \varepsilon_{0}$. 
Remark 1. Our method also works for the generalized KadomtsevPetviashvili equations

$$
u_{t}+u_{x x x}+\sigma \partial_{x}^{-1} u_{y y}=-(f(u))_{x}
$$

where $f(u)=\lambda u^{2}+\mu u^{3}, \lambda, \mu \in \boldsymbol{R}$. The same result as in Theorem 1 is true for this case.

Remark 2. If we replace $u^{2}$ by $a(t) u^{2}$ with

$$
\begin{aligned}
a(t) & \in C^{1}(\boldsymbol{R}), \quad|a(t)| \leq C(\log (1+|t|))^{-1-\gamma}, \\
\left|a^{\prime}(t)\right| & \leq C(1+|t|)^{-1}(\log (1+|t|))^{-1-\gamma},
\end{aligned}
$$

for some $\gamma>0$, then under the same conditions on the initial data as in Theorem 1 we find that there exists a unique global in time solution of the Cauchy problem for the Kadomtsev-Petviashvili equation (1.1). This fact implies that there is a possibility to prove a global existence in time of solutions to the generalized Kadomtsev-Petviashvili equation with $-\partial_{x}\left(u^{2+\delta}\right), \delta>0$ if we can consider the problem in lower order Soboev spaces of order $1+\delta$. However our method in this paper requires the Soboev spaces of order 3 .

\section{Preliminaries}

First we state the estimates for the free KP evolution group $\mathscr{U}(t)$.

Lemma 2. Let $\phi \in \boldsymbol{L}^{2} \cap \boldsymbol{L}^{q}, 1 \leq q \leq 2$. Then the estimate is true

$$
\|\mathscr{U}(t) \phi\|_{\boldsymbol{L}^{p}} \leq C|t|^{2 / p-1}\|\phi\|_{\boldsymbol{L}^{q}}
$$

for all $t \neq 0$, where $1 / p+1 / q=1$.

Lemma 2 was proved in paper [7] under the assumption that $\phi \in \boldsymbol{L}^{2} \cap \boldsymbol{L}^{q}$, $\partial_{x}^{-1} \phi \in \boldsymbol{L}^{2}$, however the condition $\partial_{x}^{-1} \phi \in \boldsymbol{L}^{2}$ can be removed, since the evolution group $\mathscr{U}(t)$ is well-defined in $\boldsymbol{L}^{2}$ (see [2]).

In the next lemma we prove the time decay estimates through the operators $\left|\mathscr{J}_{x}\right|^{\alpha}=\mathscr{U}(t)|x|^{\alpha} \mathscr{U}(-t)$ and $\left|\mathscr{J}_{y}\right|^{\beta}=\mathscr{U}(t)|y|^{\beta} \mathscr{U}(-t)$.

Lemma 3. The estimate is true

$$
\|\phi\|_{\boldsymbol{L}^{p}} \leq C t^{2 / p-1}\left(\|\phi\|_{\boldsymbol{L}^{2}}+C\left\|\left|\mathscr{J}_{x}\right|^{\alpha} \phi\right\|_{\boldsymbol{L}^{2}}+C\left\|\left|\mathscr{J}_{y}\right|^{\beta} \phi\right\|_{\boldsymbol{L}^{2}}\right)
$$

for all $t>0$, where $\alpha, \beta>0,1 / \alpha+1 / \beta<2 p /(p-2), 2 \leq p \leq \infty$, provided that the right-hand side is finite.

Proof. By the Hölder inequality with $r=2 p /(p-2), \quad 2 \leq p \leq \infty$, $1 / q+1 / p=1$ we find 


$$
\begin{aligned}
\|\psi\|_{\boldsymbol{L}^{q}} & =\left\|\left(1+|x|^{\alpha}+|y|^{\beta}\right)^{-1}\left(1+|x|^{\alpha}+|y|^{\beta}\right) \psi\right\|_{\boldsymbol{L}^{q}} \\
& \leq\left\|\left(1+|x|^{\alpha}+|y|^{\beta}\right)^{-1}\right\|_{\boldsymbol{L}^{r}}\left\|\left(1+|x|^{\alpha}+|y|^{\beta}\right) \psi\right\|_{\boldsymbol{L}^{2}} \\
& \leq C\|\psi\|_{\boldsymbol{L}^{2}}+C\left\||x|^{\alpha} \psi\right\|_{\boldsymbol{L}^{2}}+C\left\||y|^{\beta} \psi\right\|_{\boldsymbol{L}^{2}},
\end{aligned}
$$

since

$$
\begin{aligned}
\left\|\left(1+|x|^{\alpha}+|y|^{\beta}\right)^{-1}\right\|_{\boldsymbol{L}^{r}}^{r} & =\iint_{\boldsymbol{R}^{2}} \frac{d x d y}{\left(1+|x|^{\alpha}+|y|^{\beta}\right)^{v}\left(1+|x|^{\alpha}+|y|^{\beta}\right)^{r-v}} \\
& \leq \int_{\boldsymbol{R}}\left(1+|x|^{\alpha}\right)^{-v} d x \int_{\boldsymbol{R}}\left(1+|y|^{\beta}\right)^{v-r} d y \leq C
\end{aligned}
$$

if we choose $1 / \alpha<v<r-1 / \beta$, and $1 / \alpha+1 / \beta<r=2 p /(p-2)$. Hence taking $\psi=\mathscr{U}(-t) \phi$, by Lemma 2 we get for $2 \leq p \leq \infty, 1 / q+1 / p=1$

$$
\begin{aligned}
\|\phi\|_{L^{p}} & =\|\mathscr{U}(t) \mathscr{U}(-t) \phi\|_{L^{p}} \\
& \leq C t^{2 / p-1}\|\mathscr{U}(-t) \phi\|_{L^{q}} \\
& \leq C t^{2 / p-1}\left(\|\phi\|_{L^{2}}+C\left\||x|^{\alpha} \mathscr{U}(-t) \phi\right\|_{L^{2}}+C\left\||y|^{\beta} \mathscr{U}(-t) \phi\right\|_{L^{2}}\right. \\
& \leq C t^{2 / p-1}\left(\|\phi\|_{L^{2}}+C\left\|\left|\mathscr{J}_{x}\right|^{\alpha} \phi\right\|_{L^{2}}+C\left\|\left|\mathscr{J}_{y}\right|^{\beta} \phi\right\|_{L^{2}}\right)
\end{aligned}
$$

for all $t>0$. This completes the proof of the lemma.

In the next lemma we prove the time decay estimates through the norm

$$
\|\phi\|_{\boldsymbol{Y}}=\left\|\partial_{y} \partial_{x}^{-1} \phi\right\|_{\boldsymbol{H}^{1}}+\|\phi\|_{\boldsymbol{H}^{1}}+\left\|\partial_{x}^{3} \phi\right\|_{\boldsymbol{L}^{2}}+\left\|\mathscr{J}_{x} \partial_{x} \phi\right\|_{\boldsymbol{L}^{2}}+\left\|\mathscr{J}_{y}^{2} \partial_{x} \phi\right\|_{\boldsymbol{L}^{2}}
$$

Lemma 4. The estimate is true

$$
\langle t\rangle^{1 / 2}\left\|\mathscr{J}_{y} \partial_{x} \phi\right\|_{L^{4}}+\langle t\rangle^{1 / 2}\left\|\partial_{y} \phi\right\|_{L^{4}}+\langle t\rangle\left\|\partial_{x} \phi\right\|_{L^{\infty}} \leq C\|\phi\|_{\boldsymbol{Y}}
$$

for all $t \in \boldsymbol{R}$, provided that the right-hand side is finite.

Proof. By Lemma 3 with $p=4, \alpha=1 / 2, \beta=1$ we find

$$
\left\|\mathscr{J}_{y} \phi\right\|_{L^{4}} \leq C t^{-1 / 2}\left(\left\|\mathscr{J}_{y} \phi\right\|_{L^{2}}+\left\|\left|\mathscr{J}_{x}\right|^{1 / 2} \mathscr{J}_{y} \phi\right\|_{L^{2}}+\left\|\left|\mathscr{J}_{y}\right| \mathscr{J}_{y} \phi\right\|_{L^{2}}\right) .
$$

By the Cauchy-Schwarz inequality we get

$$
\begin{aligned}
\left\|\mathscr{J}_{y} \phi\right\|_{L^{2}}^{2} & =\left(\mathscr{U}(-t) \phi, y^{2} \mathscr{U}(-t) \phi\right) \\
& \leq\|\phi\|_{\boldsymbol{L}^{2}}\left\|y^{2} \mathscr{U}(-t) \phi\right\|_{\boldsymbol{L}^{2}}=\|\phi\|_{\boldsymbol{L}^{2}}\left\|\mathscr{J}_{y}^{2} \phi\right\|_{\boldsymbol{L}^{2}} \\
& \leq\|\phi\|_{\boldsymbol{L}^{2}}^{2}+\left\|\mathscr{J}_{y}^{2} \phi\right\|_{\boldsymbol{L}^{2}}^{2} .
\end{aligned}
$$


In the same manner

$$
\begin{aligned}
\left\|\left|\mathscr{J}_{x}\right|^{1 / 2} \mathscr{J}_{y} \phi\right\|_{L^{2}}^{2} & =\left\||x|^{1 / 2} y \mathscr{U}(-t) \phi\right\|_{L^{2}}^{2}=\left(|x| \mathscr{U}(-t) \phi, y^{2} \mathscr{U}(-t) \phi\right) \\
& \leq\|x \mathscr{U}(-t) \phi\|_{L^{2}}\left\|y^{2} \mathscr{U}(-t) \phi\right\|_{L^{2}}=\left\|\mathscr{J}_{x} \phi\right\|_{L^{2}}\left\|\mathscr{J}_{y}^{2} \phi\right\|_{L^{2}} \\
& \leq\left\|\mathscr{J}_{x} \phi\right\|_{\boldsymbol{L}^{2}}^{2}+\left\|\mathscr{J}_{y}^{2} \phi\right\|_{\boldsymbol{L}^{2}}^{2} .
\end{aligned}
$$

Also

$$
\left\|\left|\mathscr{J}_{y}\right| \mathscr{J}_{y} \phi\right\|_{\boldsymbol{L}^{2}}=\||y| y \mathscr{U}(-t) \phi\|_{\boldsymbol{L}^{2}}=\left\|y^{2} \mathscr{U}(-t) \phi\right\|_{\boldsymbol{L}^{2}}=\left\|\mathscr{J}_{y}^{2} \phi\right\|_{\boldsymbol{L}^{2}}
$$

Therefore we obtain

$$
\left\|\mathscr{J}_{y} \partial_{x} \phi\right\|_{\boldsymbol{L}^{4}} \leq C t^{-1 / 2}\left(\left\|\partial_{x} \phi\right\|_{L^{2}}+\left\|\mathscr{J}_{x} \partial_{x} \phi\right\|_{\boldsymbol{L}^{2}}+\left\|\mathscr{J}_{y}^{2} \partial_{x} \phi\right\|_{\boldsymbol{L}^{2}}\right)
$$

for all $t \geq 1$. On the other hand, by the Sobolev imbedding theorem $\|\phi\|_{L^{4}} \leq$ $C\left\|(-\Delta)^{1 / 4} \phi\right\|_{L^{2}}$ (see [9]) we find

$$
\left\|\mathscr{J}_{y} \partial_{x} \phi\right\|_{L^{4}} \leq C\left\|\left|\partial_{x}\right|^{1 / 2} \mathscr{J}_{y} \partial_{x} \phi\right\|_{L^{2}}+C\left\|\left|\partial_{y}\right|^{1 / 2} \mathscr{J}_{y} \partial_{x} \phi\right\|_{L^{2}}
$$

We use the Plancherel theorem and the integration by parts to see that

$$
\begin{aligned}
\left\|\left|\partial_{x}\right|^{1 / 2} \mathscr{J}_{y} \partial_{x} \phi\right\|_{L^{2}}^{2} & =\left|\left(\mathscr{J}_{y}^{2} \partial_{x} \phi,\left|\partial_{x}\right| \partial_{x} \phi\right)\right| \\
& \leq C\left\|\mathscr{J}_{y}^{2} \partial_{x} \phi\right\|_{L^{2}}\left\|\partial_{x}^{2} \phi\right\|_{L^{2}} \\
& \leq C\left\|\mathscr{J}_{y}^{2} \partial_{x} \phi\right\|_{L^{2}}\left\|\partial_{x} \phi\right\|_{L^{2}}^{1 / 2}\left\|\partial_{x}^{3} \phi\right\|_{L^{2}}^{1 / 2} .
\end{aligned}
$$

In the same way, integration by parts yields

$$
\begin{aligned}
\left\|\left|\partial_{y}\right|^{1 / 2} \mathscr{J}_{y} \partial_{x} \phi\right\|_{\boldsymbol{L}^{2}}^{2}= & \left\|\left|\partial_{y}\right|^{1 / 2} y \mathscr{U}(-t) \partial_{x} \phi\right\|_{\boldsymbol{L}^{2}}^{2} \\
= & \left|\left(\mathscr{F} \mathscr{U}(-t) \partial_{x} \phi, \partial_{\eta}|\eta| \partial_{\eta} \mathscr{F} \mathscr{U}(-t) \partial_{x} \phi\right)\right| \\
\leq & \left|\left(|\eta| \mathscr{F} \mathscr{U}(-t) \partial_{x} \phi, \partial_{\eta}^{2} \mathscr{F} \mathscr{U}(-t) \partial_{x} \phi\right)\right| \\
& +\left|\left(\mathscr{F} \mathscr{U}(-t) \partial_{x} \phi, \eta|\eta|^{-1} \partial_{\eta} \mathscr{F} \mathscr{U}(-t) \partial_{x} \phi\right)\right| \\
\leq & \left\|\partial_{y} \partial_{x} \phi\right\|_{\boldsymbol{L}^{2}}\left\|\mathscr{J}_{y}^{2} \partial_{x} \phi\right\|_{\boldsymbol{L}^{2}}+\left\|\partial_{x} \phi\right\|_{\boldsymbol{L}^{2}}\left\|\mathscr{J}_{y} \partial_{x} \phi\right\|_{\boldsymbol{L}^{2}} \\
\leq & \left\|\partial_{y}^{2} \partial_{x}^{-1} \phi\right\|_{\boldsymbol{L}^{2}}^{1 / 2}\left\|\partial_{x}^{3} \phi\right\|_{\boldsymbol{L}^{2}}^{1 / 2}\left\|\mathscr{J}_{y}^{2} \partial_{x} \phi\right\|_{\boldsymbol{L}^{2}}+\left\|\partial_{x} \phi\right\|_{\boldsymbol{L}^{2}}^{3 / 2}\left\|\mathscr{J}_{y}^{2} \partial_{x} \phi\right\|_{\boldsymbol{L}^{2}}^{1 / 2} .
\end{aligned}
$$

Therefore

$$
\left\|\mathscr{J}_{y} \partial_{x} \phi\right\|_{L^{4}} \leq C\left(\left\|\partial_{x}^{-1} \phi\right\|_{H^{2}}+\left\|\partial_{x}^{3} \phi\right\|_{L^{2}}+\left\|\mathscr{J}_{y}^{2} \partial_{x} \phi\right\|_{L^{2}}\right)
$$

for all $t \geq 0$. Combining estimates (2.2) and (2.3) we obtain the first estimate of the lemma. By Lemma 3 with $p=4, \alpha=1 / 2, \beta=1$ we find

$$
\left\|\partial_{y} \phi\right\|_{\boldsymbol{L}^{4}} \leq C t^{-1 / 2}\left(\left\|\partial_{y} \phi\right\|_{\boldsymbol{L}^{2}}+\left\|\left|\mathscr{J}_{x}\right|^{1 / 2} \partial_{y} \phi\right\|_{\boldsymbol{L}^{2}}+\left\|\left|\mathscr{J}_{y}\right| \partial_{y} \phi\right\|_{\boldsymbol{L}^{2}}\right) .
$$


By the Cauchy-Schwarz inequality and integration by parts we get

$$
\begin{aligned}
\left\|\left|\mathscr{J}_{x}\right|^{1 / 2} \partial_{y} \phi\right\|_{L^{2}}^{2} & =\left\||x|^{1 / 2} \mathscr{U}(-t) \partial_{y} \phi\right\|_{L^{2}}^{2} \\
& =-\left(|x| \mathscr{U}(-t) \phi, \mathscr{U}(-t) \partial_{y}^{2} \phi\right)=\left(\partial_{x}|x| \mathscr{U}(-t) \phi, \mathscr{U}(-t) \partial_{x}^{-1} \partial_{y}^{2} \phi\right) \\
& \leq\left(\|\phi\|_{L^{2}}+\left\|\mathscr{J}_{x} \partial_{x} \phi\right\|_{L^{2}}\right)\left\|\partial_{x}^{-1} \partial_{y}^{2} \phi\right\|_{L^{2}}
\end{aligned}
$$

In the same way

$$
\begin{aligned}
\left\|\left|\mathscr{J}_{y}\right| \partial_{y} \phi\right\|_{\boldsymbol{L}^{2}}^{2} & =\left\||y| \mathscr{U}(-t) \partial_{y} \phi\right\|_{\boldsymbol{L}^{2}}^{2} \\
& =-\left(|y|^{2} \mathscr{U}(-t) \phi, \mathscr{U}(-t) \partial_{y}^{2} \phi\right)-2\left(y \mathscr{U}(-t) \phi, \mathscr{U}(-t) \partial_{y} \phi\right) \\
& =\left(|y|^{2} \mathscr{U}(-t) \partial_{x} \phi, \mathscr{U}(-t) \partial_{x}^{-1} \partial_{y}^{2} \phi\right)+2\left(y \mathscr{U}(-t) \partial_{x} \phi, \mathscr{U}(-t) \partial_{x}^{-1} \partial_{y} \phi\right) \\
& \leq\left\|\mathscr{J}_{y}^{2} \partial_{x} \phi\right\|_{L^{2}}\left\|\partial_{x}^{-1} \partial_{y}^{2} \phi\right\|_{L^{2}}+2\left\|\mathscr{J}_{y} \partial_{x} \phi\right\|_{\boldsymbol{L}^{2}}\left\|\partial_{x}^{-1} \partial_{y} \phi\right\|_{L^{2}}
\end{aligned}
$$

Therefore by (2.1) we obtain

$$
\left\|\partial_{y} \phi\right\|_{\boldsymbol{L}^{4}} \leq C t^{-1 / 2}\left(\left\|\partial_{x}^{-1} \partial_{y} \phi\right\|_{\boldsymbol{H}^{1}}+\|\phi\|_{\boldsymbol{H}^{1}}+\left\|\mathscr{J}_{x} \partial_{x} \phi\right\|_{\boldsymbol{L}^{2}}+\left\|\mathscr{J}_{y}^{2} \partial_{x} \phi\right\|_{\boldsymbol{L}^{2}}\right)
$$

for all $t \geq 1$. On the other hand, by the Sobolev imbedding theorem we get

$$
\left\|\partial_{y} \phi\right\|_{L^{4}} \leq C\left\|\left|\partial_{x}\right|^{1 / 2} \partial_{y} \phi\right\|_{L^{2}}+C\left\|\left|\partial_{y}\right|^{1 / 2} \partial_{y} \phi\right\|_{L^{2}}
$$

By the Cauchy-Schwarz inequality, the Plancherel theorem and the integration by parts we get

$$
\begin{aligned}
\left\|\left|\partial_{x}\right|^{1 / 2} \partial_{y} \phi\right\|_{\boldsymbol{L}^{2}}^{2} & =\left(\partial_{y} \phi,\left|\partial_{x}\right| \partial_{y} \phi\right)=\left(\partial_{x}^{-1} \partial_{y}^{2} \phi, \partial_{x}\left|\partial_{x}\right| \phi\right) \\
& \leq C\left\|\partial_{x}^{-1} \partial_{y}^{2} \phi\right\|_{\boldsymbol{L}^{2}}\left\|\partial_{x}\left|\partial_{x}\right| \phi\right\|_{\boldsymbol{L}^{2}} \\
& \leq C\left\|\partial_{x}^{-1} \partial_{y}^{2} \phi\right\|_{\boldsymbol{L}^{2}}\left\|\partial_{x} \phi\right\|_{\boldsymbol{L}^{2}}^{1 / 2}\left\|\partial_{x}^{3} \phi\right\|_{\boldsymbol{L}^{2}}^{1 / 2}
\end{aligned}
$$

since $\partial_{x} \partial_{x}^{-1}=\partial_{x} \int_{-\infty}^{x} d x^{\prime}=I$. In the same way, integration by parts yields

$$
\begin{aligned}
\left\|\left|\partial_{y}\right|^{1 / 2} \partial_{y} \phi\right\|_{L^{2}}^{2} & =\left(\left|\partial_{y}\right| \partial_{y} \phi, \partial_{y} \phi\right)=-\left(\left|\partial_{y}\right| \partial_{y} \partial_{x}^{-1} \phi, \partial_{x} \partial_{y} \phi\right) \\
& \leq\left\|\partial_{x}^{-1} \partial_{y}^{2} \phi\right\|_{L^{2}}\left\|\partial_{x} \partial_{y} \phi\right\|_{L^{2}} \\
& \leq\left\|\partial_{x}^{-1} \partial_{y}^{2} \phi\right\|_{L^{2}}^{3 / 2}\left\|\partial_{x}^{3} \phi\right\|_{L^{2}}^{1 / 2}
\end{aligned}
$$

Therefore

$$
\left\|\mathscr{J}_{y} \partial_{x} \phi\right\|_{\boldsymbol{L}^{4}} \leq C\left(\left\|\partial_{y} \partial_{x}^{-1} \phi\right\|_{\boldsymbol{H}^{1}}+\|\phi\|_{\boldsymbol{H}^{1}}+\left\|\partial_{x}^{3} \phi\right\|_{\boldsymbol{L}^{2}}\right)
$$


for all $t \geq 0$. Combining estimates (2.4) and (2.5) we obtain the second estimate of the lemma. By Lemma 3 with $p=\infty, \alpha=1, \beta=2$ we find

$$
\left\|\partial_{x} \phi\right\|_{\boldsymbol{L}^{\infty}} \leq C t^{-1}\left(\left\|\partial_{x} \phi\right\|_{\boldsymbol{L}^{2}}+\left\|\mathscr{J}_{x} \partial_{x} \phi\right\|_{\boldsymbol{L}^{2}}+\left\|\mathscr{J}_{y}^{2} \partial_{x} \phi\right\|_{\boldsymbol{L}^{2}}\right)
$$

for all $t \geq 1$. On the other hand, by the Cauchy-Schwarz inequality and the Plancherel theorem we have

$$
\begin{aligned}
\left\|\partial_{x} \phi\right\|_{L^{\infty}} & \leq C\left\|\mathscr{F} \partial_{x} \phi\right\|_{L^{1}} \\
& \leq C\left\|\left(1+|\xi|^{2}+|\eta|\right)^{-1}\right\|_{L^{2}}\left\|\left(1+|\xi|^{2}+|\eta|\right) \mathscr{F} \partial_{x} \phi\right\|_{L^{2}} \\
& \leq C\left\|\partial_{x} \phi\right\|_{L^{2}}+C\left\|\partial_{y} \partial_{x} \phi\right\|_{L^{2}}+C\left\|\partial_{x}^{3} \phi\right\|_{L^{2}} \\
& \leq C\left\|\partial_{x} \phi\right\|_{L^{2}}+C\left\|\partial_{y}^{2} \partial_{x}^{-1} \phi\right\|_{L^{2}}+C\left\|\partial_{x}^{3} \phi\right\|_{L^{2}}
\end{aligned}
$$

which gives us the third estimate of the lemma. Lemma 4 is proved.

\section{Proof of Theorem 1}

We need to estimate the norms $\left\|\partial_{y} \partial_{x}^{-1} u\right\|_{H^{1}},\|u\|_{H^{1}},\left\|\partial_{x}^{3} u\right\|_{L^{2}},\left\|\mathscr{J}_{y}^{2} \partial_{x} u\right\|_{L^{2}}$ and $\left\|\mathscr{J}_{x} \partial_{x} u\right\|_{\boldsymbol{L}^{2}}$. Multiplying equation (1.1) by $\partial_{y}^{2} \partial_{x}^{-1}, \partial_{y} \partial_{x}^{-1}, \partial_{x}, \partial_{y}$ and $\partial_{x}^{3}$, applying the energy method and integrating by parts we get

$$
\begin{aligned}
\frac{d}{d t}\left(\left\|\partial_{y} \partial_{x}^{-1} u\right\|_{\boldsymbol{H}^{1}}+\|u\|_{\boldsymbol{H}^{1}}+\left\|\partial_{x}^{3} u\right\|_{\boldsymbol{L}^{2}}\right) \\
\leq C\left\|\partial_{x} u\right\|_{\boldsymbol{L}^{\infty}}\left(\left\|\partial_{y} \partial_{x}^{-1} u\right\|_{\boldsymbol{H}^{2}}+\|u\|_{\boldsymbol{H}^{1}}+\left\|\partial_{x}^{3} u\right\|_{\boldsymbol{L}^{2}}\right) \\
\quad+C\left\|\partial_{x}^{2} u\right\|_{\boldsymbol{L}^{4}}^{2}+C\left\|\partial_{y} u\right\|_{\boldsymbol{L}^{4}}^{2} \\
\leq C\langle t\rangle^{-1}\|u\|_{\boldsymbol{Y}}^{2}
\end{aligned}
$$

since $\left\|\partial_{x}^{2} u\right\|_{L^{4}}^{2} \leq C\left\|\partial_{x} u\right\|_{L^{\infty}}\left\|\partial_{x}^{3} u\right\|_{L^{2}}$ and by Lemma 4 we have the estimates $\left\|\partial_{x} u\right\|_{L^{\infty}} \leq C\langle t\rangle^{-1}\|u\|_{\boldsymbol{Y}},\left\|\partial_{y} u\right\|_{L^{4}}^{2} \leq C\langle t\rangle^{-1}\|u\|_{\boldsymbol{Y}}^{2}$. We next multiply equation (1.1) by the operator $\mathscr{J}_{y}^{2} \partial_{x}$

$$
\mathscr{L}_{y}^{2} \partial_{x} u=-2\left(\mathscr{J}_{y} \partial_{x} u\right)^{2}-2 u \partial_{x}\left(\mathscr{J}_{y}^{2} \partial_{x} u\right) .
$$

Then multiplying equation (3.2) by $\mathscr{J}_{y}^{2} \partial_{x} u$ and integrating by parts with respect to $x$, we obtain

$$
\begin{aligned}
\frac{d}{d t}\left\|\mathscr{J}_{y}^{2} \partial_{x} u\right\|_{\boldsymbol{L}^{2}} & \leq C\left\|\mathscr{J}_{y} \partial_{x} u\right\|_{\boldsymbol{L}^{4}}^{2}+C\left\|\partial_{x} u\right\|_{\boldsymbol{L}^{\infty}}\left\|\mathscr{J}_{y}^{2} \partial_{x} u\right\|_{\boldsymbol{L}^{2}} \\
& \leq C\langle t\rangle^{-1}\|u\|_{\boldsymbol{Y}}^{2}
\end{aligned}
$$


since by Lemma 4 we have the estimates $\left\|\mathscr{J}_{y} \partial_{x} u\right\|_{L^{4}} \leq C\langle t\rangle^{-1 / 2}\|u\|_{Y}$ and $\left\|\partial_{x} u\right\|_{L^{\infty}} \leq C\langle t\rangle^{-1}\|u\|_{\boldsymbol{Y}}$. Integrating (3.1) and (3.3) with respect to $t$ we obtain

$$
\begin{aligned}
& \left\|\partial_{y} \partial_{x}^{-1} u\right\|_{\boldsymbol{H}^{1}}+\|u\|_{\boldsymbol{H}^{1}}+\left\|\partial_{x}^{3} u\right\|_{\boldsymbol{L}^{2}}+\left\|\mathscr{J}_{y}^{2} \partial_{x} u\right\|_{\boldsymbol{L}^{2}} \\
& \leq\left\|\partial_{y} \partial_{x}^{-1} u_{0}\right\|_{\boldsymbol{H}^{1}}+\left\|u_{0}\right\|_{\boldsymbol{H}^{1}}+\left\|\partial_{x}^{3} u_{0}\right\|_{\boldsymbol{L}^{2}}+\left\|y^{2} \partial_{x} u_{0}\right\|_{\boldsymbol{L}^{2}} \\
& \quad+C \int_{0}^{t}\langle\tau\rangle^{-1}\|u\|_{\boldsymbol{Y}}^{2} d \tau
\end{aligned}
$$

It seems to be difficult to estimate $\mathscr{J}_{x} \partial_{x} u$ directly by equation (1.1). Instead we use the first order operator $\mathscr{P}=x \partial_{x}+2 y \partial_{y}+3 t \partial_{t}$, since the identity $\mathscr{J}_{x} \partial_{x}=\mathscr{P}-2 \mathscr{J}_{y} \partial_{y}-3 t \mathscr{L}$ is true. We apply the first order operator $\mathscr{P}$ to equation (1.1), then in view of the commutator relations $[\mathscr{L}, \mathscr{P}]=3 \mathscr{L}$ and $\left[\partial_{x}, \mathscr{P}\right]=\partial_{x}$, we obtain

$$
\mathscr{L} \mathscr{P} u=-2 u \partial_{x} \mathscr{P} u-2\left(\partial_{x} u\right) \mathscr{P} u-4 u \partial_{x} u
$$

Multiplying (3.5) by $\mathscr{P} u$, integrating in space, we get

$$
\frac{d}{d t}\|\mathscr{P} u\|_{L^{2}} \leq C\left\|\partial_{x} u\right\|_{L^{\infty}}\left(\|\mathscr{P} u\|_{L^{2}}+\|u\|_{L^{2}}\right)
$$

By the identity $\mathscr{P}=\mathscr{J}_{x} \partial_{x}+2 \mathscr{J}_{y} \partial_{y}+3 t \mathscr{L}$ and inequality

$$
\begin{aligned}
\left\|\mathscr{J}_{y} \partial_{y} u\right\|_{\boldsymbol{L}^{2}}^{2} & =\left|\left(\mathscr{J}_{y}^{2} \partial_{y} \partial_{x} u, \partial_{y} \partial_{x}^{-1} u\right)\right|=\left|\left(\mathscr{J}_{y}^{2} \partial_{x} u, \partial_{y}^{2} \partial_{x}^{-1} u\right)+2\left(\mathscr{J}_{y} \partial_{x} u, \partial_{y} \partial_{x}^{-1} u\right)\right| \\
& \leq\left\|\mathscr{J}_{y}^{2} \partial_{x} u\right\|_{L^{2}}\left\|\partial_{y}^{2} \partial_{x}^{-1} u\right\|_{\boldsymbol{L}^{2}}+2\left\|\mathscr{J}_{y} \partial_{x} u\right\|_{\boldsymbol{L}^{2}}\left\|\partial_{y} \partial_{x}^{-1} u\right\|_{\boldsymbol{L}^{2}} \\
& \leq\left\|\mathscr{J}_{y}^{2} \partial_{x} u\right\|_{\boldsymbol{L}^{2}}\left\|\partial_{y} \partial_{x}^{-1} u\right\|_{\boldsymbol{H}^{1}}+2\left\|\mathscr{J}_{y}^{2} \partial_{x} u\right\|_{\boldsymbol{L}^{2}}^{1 / 2}\left\|\partial_{x} u\right\|_{\boldsymbol{L}^{2}}^{1 / 2}\left\|\partial_{y} \partial_{x}^{-1} u\right\|_{\boldsymbol{L}^{2}} \\
& \leq 4\left(\left\|\partial_{y} \partial_{x}^{-1} u\right\|_{\boldsymbol{H}^{1}}+\left\|\partial_{x} u\right\|_{\boldsymbol{L}^{2}}+\left\|\mathscr{J}_{y}^{2} \partial_{x} u\right\|_{\boldsymbol{L}^{2}}\right)^{2}
\end{aligned}
$$

it follows that

$$
\left\|\mathscr{J}_{x} \partial_{x} u\right\|_{\boldsymbol{L}^{2}} \leq\|\mathscr{P} u\|_{\boldsymbol{L}^{2}}+4\left\|\partial_{y} \partial_{x}^{-1} u\right\|_{\boldsymbol{H}^{1}}+4\|u\|_{\boldsymbol{H}^{1}}+4\left\|\mathscr{J}_{y}^{2} \partial_{x} u\right\|_{\boldsymbol{L}^{2}}+C\|u\|_{\boldsymbol{Y}}^{2}
$$

and

$$
\|\mathscr{P} u\|_{L^{2}} \leq C\|u\|_{\boldsymbol{Y}}\left(1+\|u\|_{\boldsymbol{Y}}\right) .
$$

Hence in view of the second estimate of Lemma 4 we find by (3.6)

$$
\frac{d}{d t}\|\mathscr{P} u\|_{L^{2}} \leq C\langle t\rangle^{-1}\|u\|_{\boldsymbol{Y}}^{2}\left(1+\|u\|_{\boldsymbol{Y}}\right) .
$$


Integrating (3.7) with respect to $t$ we obtain

$$
\begin{aligned}
\left\|\mathscr{J}_{x} \partial_{x} u\right\|_{\boldsymbol{L}^{2}} \leq & \|\mathscr{P} u\|_{\boldsymbol{L}^{2}}+4\left\|\partial_{y} \partial_{x}^{-1} u\right\|_{\boldsymbol{H}^{1}}+4\|u\|_{\boldsymbol{H}^{1}}+4\left\|\mathscr{J}_{y}^{2} \partial_{x} u\right\|_{\boldsymbol{L}^{2}}+C\|u\|_{\boldsymbol{Y}}^{2} \\
\leq & \|\mathscr{P} u(0)\|_{\boldsymbol{L}^{2}}+4\left\|\partial_{y} \partial_{x}^{-1} u\right\|_{\boldsymbol{H}^{1}}+4\|u\|_{\boldsymbol{H}^{1}}+4\left\|\mathscr{J}_{y}^{2} \partial_{x} u\right\|_{\boldsymbol{L}^{2}}+C\|u\|_{\boldsymbol{Y}}^{2} \\
& +C \int_{0}^{t}\langle\tau\rangle^{-1}\|u\|_{\boldsymbol{Y}}^{2}\left(1+\|u\|_{\boldsymbol{Y}}\right) d \tau
\end{aligned}
$$

from which and (3.4) it follows that

$$
\begin{aligned}
\|u\|_{\boldsymbol{Y}} & \leq \epsilon+C\|u\|_{\boldsymbol{X}_{T}}^{2}+C \int_{0}^{t}\langle\tau\rangle^{-1}\|u\|_{\boldsymbol{X}_{T}}^{2}\left(1+\|u\|_{\boldsymbol{X}_{T}}\right) d \tau \\
& \leq \epsilon+C\|u\|_{\boldsymbol{X}_{T}}^{2}\left(1+\|u\|_{\boldsymbol{X}_{T}}\right) \log (1+T)
\end{aligned}
$$

where

$$
\begin{aligned}
\epsilon & =\|\mathscr{P} u(0)\|_{\boldsymbol{L}^{2}}+5\left\|\partial_{y} \partial_{x}^{-1} u_{0}\right\|_{\boldsymbol{H}^{1}}+5\left\|u_{0}\right\|_{\boldsymbol{H}^{1}}+5\left\|\partial_{x}^{3} u_{0}\right\|_{\boldsymbol{L}^{2}}+5\left\|y^{2} \partial_{x} u_{0}\right\|_{\boldsymbol{L}^{2}} \\
& \leq 6\left\|u_{0}\right\|_{\boldsymbol{X}_{0}} .
\end{aligned}
$$

Therefore by (3.8) we get

$$
\|u\|_{\boldsymbol{X}_{T}} \leq 6\left\|u_{0}\right\|_{\boldsymbol{X}_{0}}+C\|u\|_{\boldsymbol{X}_{T}}^{2}\left(1+\|u\|_{\boldsymbol{X}_{T}}\right) \log (1+T) .
$$

Denote

$$
T_{\varepsilon}^{*}=\sup \left\{T \in\left(0, T_{\varepsilon}\right) ;\|u\|_{X_{T}} \leq A\left\|u_{0}\right\|_{X_{0}}=A \varepsilon\right\} .
$$

We assume that

$$
\varepsilon \log \left(1+T_{\varepsilon}^{*}\right) \leq B
$$

Then by (3.9) we obtain

$$
\begin{aligned}
\|u\|_{X_{T}} & \leq 6 \varepsilon+C A^{2} B(1+A \varepsilon) \varepsilon \\
& \leq 6 \varepsilon+\frac{1}{4} \varepsilon+\frac{1}{4} A \varepsilon^{2} \leq \frac{1}{2} A \varepsilon
\end{aligned}
$$

for any $T \in\left(0, T_{\varepsilon}\right)$, if we choose $A \geq 25, B \leq\left(4 A^{2} C\right)^{-1}, \varepsilon \in\left(0, \varepsilon_{0}\right), \varepsilon_{0} \leq 1$. Therefore we find that $T_{\varepsilon}^{*}=T_{\varepsilon}$, and we can extend the solution beyond the time $t=T_{\varepsilon}^{*}$. We arrive to a contradiction. So we have

$$
T_{\varepsilon}^{*} \geq \exp \left(\frac{B}{\varepsilon}\right)-1 \geq \exp \left(\frac{B_{1}}{\varepsilon}\right) .
$$

This completes the proof of Theorem 1 . 
Acknowledgement. We would like to thank a referee for useful comments. The work of P.I.N. is partially supported by CONACYT and PAPIIT.

\section{References}

[1] de Bouard, A. and Saut, J. C., Solitary waves of generalized Kadomtsev-Petviashvili equations, Ann. Inst. H. Poincaré Anal. Non Linéaire, 14 (1997), 211-236.

[2] Bourgain, J., On the Cauchy problem for the Kadomtsev-Petviashvili equations, Geom. Funct. Anal., 3 (1993), 315-341.

[ 3 ] Hayashi, N., Naumkin, P. I. and Saut, J. C., Asymptotics for large time of global solutions to the generalized Kadomtsev-Petviashvili equation, Comm. Math. Phys., 201 (1999), 577-590.

[4] Iòrio, R. J. and Leite Nunes, W. V., On equations of KP-type, Proc. Roy. Soc. Edinburgh Sect. A, 128 (1998), 725-743.

[5] Kadmotsev, B. B. and Petviashvili, V. I., On the stability of solitary waves in weakly dispersive media, Soviet Phys. Dokl., 15 (1970), 539-541.

[6] Niizato, T., Large time behavior of solutions for the generalized Kadomtsev-Petviashvili equation, Differ. Equ. Appl., 3 (2011), 299-308.

[ 7 ] Saut, J. C., Remarks on the generalized Kadomtsev-Petviashvili equations, Indiana Univ. Math. J., 42 (1993), 1011-1026.

[ 8 ] Saut, J. C., Recent results on the generalized Kadomtsev-Petviashvili equations, Acta Appl. Math., 39 (1995), 477-487.

[9] Stein, E. M., Singular Integrals and Differentiability Properties of Functions, Princeton Mathematical Series, No. 30, Princeton Univ. Press, Princeton, NJ, 1970.

[10] Tom, M. M., On a generalized Kadomtsev-Petviashvili equation, Contemp. Math., 200, Amer. Math. Soc., Providence, RI, 1996, pp. 193-210.

[11] Turisyn, S. K. and Fal'kovitch, G. E., Stability of magnetoelastic solutions and self-focusing of sound in antiferromagnet, Soviet Phys. JETP, 62 (1985), 146-152.

[12] Ukai, S., Local solutions of the Kadomtsev-Petviashvili equation, J. Fac. Sci. Univ. Tokyo Sect. IA Math., 36 (1989), 193-209.

[13] Zhou, X., Inverse scattering transform for the time dependent Schrödinger equation with applications to the KP-I equation, Comm. Math. Phys., 128 (1990), 551-564.

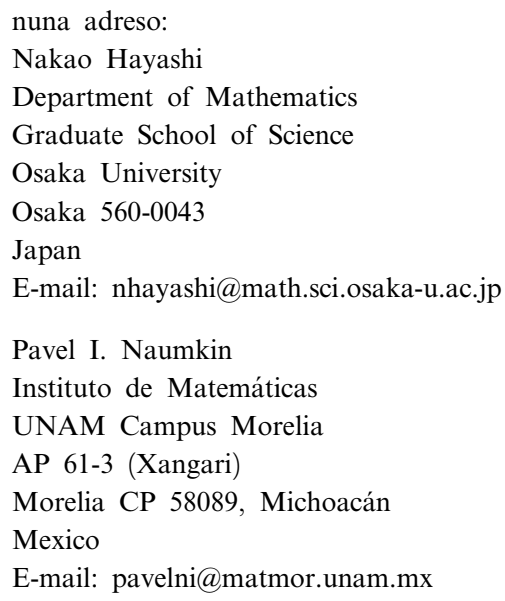




\author{
Tomoyuki Niizato \\ Department of Mathematics \\ Graduate School of Science \\ Osaka University \\ Osaka 560-0043 \\ Japan \\ E-mail: t-niizato@cr.math.sci.osaka-u.ac.jp
}

(Ricevita la 7-an de majo, 2011)

(Reviziita la 27-an de julio, 2011) 